\title{
Psicoballet en personas con diversidad funcional
}

\section{Psychoballet in people with functional diversity}

\author{
Psicoballet y diversidad funcional
}

\author{
Hernán Cedeño Cedeño. ${ }^{(1)}$ \\ Gema Zambrano Rodríguez. ${ }^{(2)}$ \\ (1) Universidad Técnica de Manabí, Portoviejo, Ecuador. email: hernanandrescedeno@gmail.com \\ (2) Universidad Técnica de Manabí, Portoviejo, Ecuador. email: gemamaria1095@gmail.com
}

Contacto: gemamaria1095@gmail.com

Recibido: 23-04-2020

Aprobado: 31-05-2020

\section{Resumen}

El objetivo de la investigación fue analizar los beneficios del psicoballet en personas con diversidad funcional en el Centro de estimulación e integración artística, Martha Terán en Portoviejo, Ecuador. La investigación es cualitativa, con un diseño fenomenológico de enfoque empírico detalló los beneficios de la técnica del psicoballet en personas con diversidad funcional. Se realizó una codificación deductiva axial de diez entrevistas semi-estructuradas y cinco grupos de enfoque; para el análisis de los datos emergentes se manipuló el software ATLAS. ti para Windows, versión 8. Los principales resultados, indican que los padres de familia, cuidadoras, maestras, directora y alumnas coinciden en que la práctica dancística aporta beneficios a nivel psíquico, biológico y social. Se concluye, que el ballet, la danza y el psicoballet, influyen positivamente en la vida de las personas con y sin ningún tipo de diversidad funcional.

Palabras clave: Psicoballet, arte, danza, ballet, diversidad funcional

\footnotetext{
Abstract

The objective of the research was to analyze the benefits of psychoballet in people with functional diversity at the Center for artistic stimulation and integration, Martha Terán in Portoviejo, Ecuador. The research is qualitative, with a phenomenological design with an empirical approach that detailed the benefits of the psychoballet technique in people with functional diversity. Axial deductive coding of ten semistructured interviews and five focus groups was performed; ATLAS software was used to analyze the emerging data. ti for Windows, version 8 . The main results indicate that parents, caregivers,
}

teachers, principal and students agree that dance practice brings benefits at the psychological, biological and social level. It is concluded that ballet, dance and psychoballet, positively influence the lives of people with and without any kind of functional diversity.

Keywords: Psychoballet, art, dance, ballet, functional diversity.

\section{Introducción}

En 1973 se crea por Georgina Fariñas un método terapéutico y cultural genuinamente cubano, recibiendo el nombre de psicoballet; mismo que en la actualidad ha sido extendido a 17 países de América Latina y Europa. De tal modo que el interes de este estudio de tipo fenomenológico se centren describir los beneficios de la técnica del psicoballet en personas con diversidad funcional.

En lo que respecta al término "diversidad funcional" este se crea en el Foro de Vida Independiente y Divertad a inicios del año 2005, mismo que fué instaurado a mediados del año 2001 a partir del objetivo por impulsar en España el movimiento de Vida Independiente, surgido en EE.UU en 1972. La terminología pretende echar abajo todas esas definiciones de tipo limitante o despectivo a propósito de "discapacidad" y en cambio reemplazarla por un significado que se ajusta a su realidad.

Romañach \& Lovato (2005), relatan lo siguiente, las mujeres y hombres con diversidad funcional somos diferentes, desde el punto de vista médico o físico, de la mayor parte de la población. Al tener características diferentes, y dadas las condiciones de entorno generadas por la sociedad, nos vemos obligados a realizar las mismas tareas o funciones de una manera diferente, algunas veces a través de 
terceras personas [...] misma función, manera diversa. (p.4)

En este sentido, el psicoballet en esencia es una modalidad de terapia artística, específicamente danzaría. Este método interrelaciona ciencia y arte de una forma armónica y balanceada. Con la psicología como base y como instrumento de acción la técnica de ballet, requiere del entrenamiento y ejecución sistemáticos que implica el desarrollo de la coordinación muscular, el control del movimiento y sentido de posición y del ritmo (Fariñas, 2011).

Para Cabezas (2013), es una terapia, porque permite un conjunto de interacciones entre un grupo de personas con problemas semejantes o que se complementan, porque que incluye un equipo conductor y porque persigue el logro de un equilibrio psíquico y social de quienes lo practican.

Fariñas (2011), afirma que se requiere, además, como en toda terapia el disfrute real de la actividad, lo cual se asegura por el trabajo artístico y creativo que se realiza" debido a la relación existente entre arte y movimiento que perfeccionan la técnica del psicoballet, logra ser posible el encuentro con la satisfacción plena de su práctica.

A todo esto vale describir lo que para Novaes el arte simboliza citado en Ballesta et al., (2011), afirma que es un bien común a todos, un quehacer esencial que acompaña al ser humano desde su origen, las actividades artísticas brindan posibilidades similares al juego para descubrir y entender el mundo desde nuestra infancia, nos permite expresar y comunicar nuestros sentimientos, emociones, pensamientos y vivencias subjetivas, así como establecer lazos entre nuestro mundo interno y el mundo exterior.

El presente trabajo tiene como objetivo analizar los beneficios del psicoballet en personas con diversidad funcional en el Centro de estimulación e integración artística, Martha Terán en Portoviejo, Ecuador.

\section{Definiciones de autores}

Rey Gónzales \& García Blas (2018) en lo referente al Trastorno De Espectro Autista (TEA) afirman: es un trastorno de origen neurobiológico que afecta a la configuración del sistema nervioso (neurodesarrollo) y al funcionamiento cerebral, dando lugar a dificultades en la comunicación y en la interacción social, así como en la flexibilidad del pensamiento y de la conducta de quien lo presenta. (p.17)
En relación al Síndrome de Down (SD), el investigador Campos (2015), relata que es causado por trisomía del gen 21 , es la anomalía cromosómica más frecuente, afectando a 1 de cada 600 a 800 recién nacidos vivos. Se caracteriza por rasgos físicos particulares, mayor riesgo de malformaciones congénitas, mayor vulnerabilidad para presentar enfermedades y discapacidad física e intelectual en grado variable. (p.101)

Por otro lado el Grupo ROP Argentina \& Ministerio de Salud (2016), revelan que la retinopatía del prematuro (ROP, acrónimo inglés de Retinopathy of Prematurity) "es una enfermedad ocular

provocada por una alteración de la vasculogénesis de la retina, que puede alterar su desarrollo normal y producir la pérdida total o parcial de la visión.

Para Gómez et al., (2013), señala que en lo referente a la Parálisis Cerebral Infantil (PCI), afirma que este síndrome su origen está localizado en el Sistema Nervioso Central (SNC), primera neurona o neurona motora superior; eso implica que casi todos los niños con PCI presentan, además de los defectos de la postura y movimiento, otros trastornos asociado" (p.30).

\section{Materiales y métodos}

Se efectuó una investigación cualitativa, con diseño fenomenológico de enfoque empírico; en el "Martha Terán-Centro de estimulación e integración artística" Portoviejo, Ecuador en los meses de julio 2019 a marzo 2020.

Se realizó una codificación deductiva - axial de diez entrevistas semi-estructuradas y cinco grupos de enfoque. "En sentido amplio, puede definirse la metodología cualitativa como la investigación que produce datos descriptivos: las propias palabras de las personas, habladas o escritas, y la conducta observable" (Quecedo Lecanda \& Castaño Garrido, 2003, p.7). Con el propósito de conceptualizar acerca de los beneficios del psicoballet, los métodos tratados abarcaron:

- Grupos de enfoque

- Entrevista semi-estructurada

- ATLAS.ti Programa de análisis cualitativo asistido por computadora (QDA).

Población y muestra: La población se compuso de 37 bailarinas y 1 bailarín, de las cuales 5 danzarinas integraban el grupo de psicoballet con edades de entre 5-12 años y diagnósticos del trastorno de espectro autista (TEA), síndrome de 
Down (SD), retinopatía del prematuro; y parálisis cerebral infantil (PCI).

En cambio, en las clases de ballet y expresión creativa estaba conformada por 16 danzarinas correspondientes al grupo de Baby-ballet con edades de entre 3-5 años y sin ningún tipo de diversidad funcional.

Las clases de ballet y danza contemporánea eran tomadas por 8 danzadoras que conformaban el grupo de infantil I con edades de entre 6-8 años, 2 bailarinas del grupo infantil II con edades de entre 9-12 años y 7 bailarinas y 1 danzante al grupo juvenil, con edades de entre 13-21 años aproximadamente y al igual que el grupo anterior no presentaban ningún tipo de diversidad funcional a excepción de una danzadora que integra el grupo de infantil

Procedimiento: En los primeros dos meses y acorde al horario establecido; se efectuó observación directa a 36 bailarinas y 1 danzante en el lugar donde se desenvolvía la investigación, faltando entre ellos una pequeña danzadora que debido a contextos personales no fue posible dicha observación.

Una vez finalizado el indicado método de recolección de datos, se adelantó al proceso de entrevistas semi-estructurada, con la directora; cuidadoras de las pequeñas con autorización de los padres y padres de familia de las bailarinas que integraban el grupo de psicoballet; a cada uno de los participantes se le formularon tres preguntas:

- ¿Cree Ud. que el psicoballet ha sido de gran ayuda para su hija?
- ¿Cuáles considera Ud. los beneficios aportados por el Psicoballet?

- ¿Cuál cree Ud. que hayan sido los logros alcanzados por el psicoballet?

Por otro lado, los representantes de las pequeñas que componían el grupo de Baby-ballet, Infantil I, e Infantil II; mediante la técnica cualitativa Grupo focal; comentaron los beneficios del ballet y la danza contemporánea evidenciados en sus hijas.

De igual modo a los jóvenes que constituían el Grupo juvenil y a la maestra de danza se les pregunto acerca de los beneficios que traía consigo la práctica del ballet y la danza contemporánea; adicional a aquello se les expuso dos preguntas: ¿Cómo describiría Ud. la danza/ballet en una sola palabra? Imagina Ud. una vida sin la danza/ballet?" (véase la figura 3).

Y por último para el tratamiento analítico de los datos se utilizó ATLAS.ti, for Windows -versión 8.

\section{Resultados}

Mediante la recolección de información en base a entrevistas semi-estructurada a los padres de familia, cuidadoras y maestra, con relación a los beneficios de la práctica del psicoballet; evidenciados en las bailarinas con algún tipo de diversidad funcional fueron desarrolladas

categorías con los siguientes porcentajes: autoestima $17 \%$, bienestar emocional $17 \%$, comunicación $6 \%$, conducta $6 \%$, desenvolvimiento escénico $12 \%$, educación artística $6 \%$, habilidades sociales $18 \%$, imaginación constructiva $6 \%$, motricidad $6 \%$ y relajación $6 \%$, se observan en el gráfico 1 .
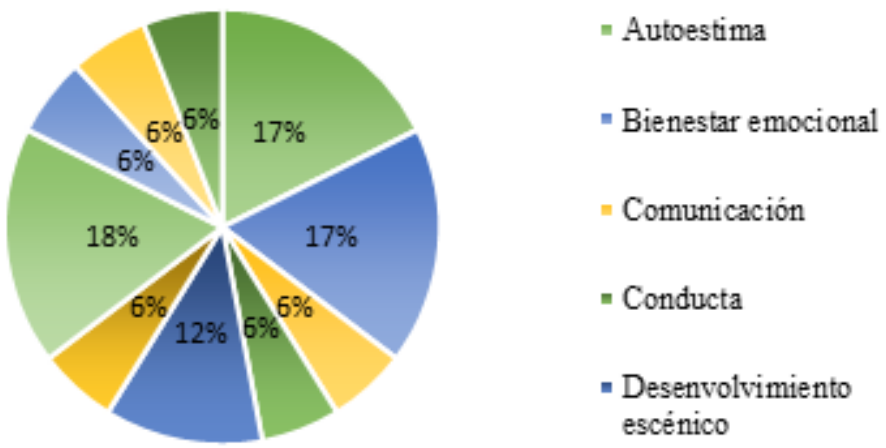

Gráfico 1. Beneficios de las clases de psicoballet.

Los resultados de las entrevistas reflejan los beneficios de las clases de ballet, en los concerniente a la expresión creativa y danza contemporánea mostraron categorías con los siguientes porcentajes: concentración 8\%, destrezas $17 \%$, disciplina $17 \%$, felicidad $17 \%$, habilidades sociales $25 \%$, independencia $8 \%$ y motricidad 8\%, gráfico Nro. 2. 


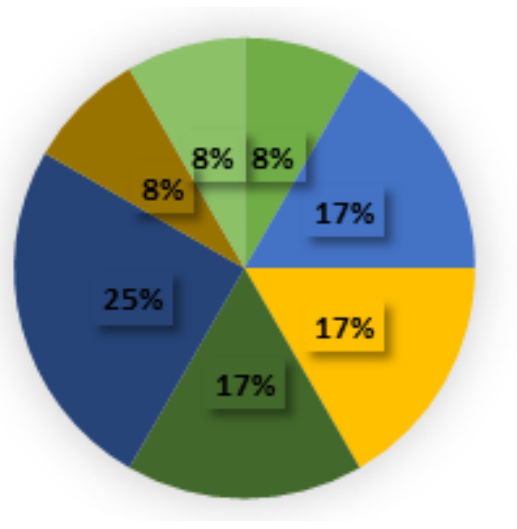

\author{
Concentración \\ -Destrezas \\ Disciplina \\ -Felicidad \\ - Habilidades sociales \\ -Independencia \\ Motricidad
}

Gráfico 2. Beneficios de las clases de ballet, expresión creativa y danza contemporánea.

En lo correspondiente a los beneficios de las clases de ballet y danza contemporánea afirmados por los participantes de la presente investigación fortalecieron categorías con los siguientes

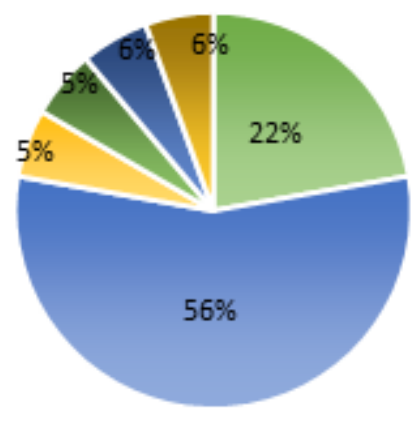

porcentajes: autonomía $22 \%$, bienestar emocional $56 \%$, conciencia corporal $5 \%$, disciplina $5 \%$, relaciones interpersonales $6 \%$ y rendimiento físico $6 \%$, se aprecian en el gráfico Nro.3.

Gráfico 3. Beneficios de las clases de ballet y danza contemporánea

Al examinar las respuestas de las entrevistas, se observó que las clases de psicoballet consolidan el bienestar emocional de sus practicantes, en el escenario que se desarrolló el estudio.

En el instrumento aplicado un estudiante de ballet, afirma: "El hecho de hacer esta actividad física llamada danza o en el caso a la vez del ballet, entrena el cuerpo; entonces el cuerpo es más capaz de hacer más cosas y de resistir más, de aguantar" (Noviembre, 2019).

Por otra parte la cuidadora de una de las bailarinas, atestigua: "Yo siento que sí le ayuda a los niños bastante a sentirse mejor, a distraerse un poco de la rutina diaria del trabajo de ellos; por ejemplo los niños con autismo en el caso tienen sus terapias, digamos el psicoballet es como un deporte para ellos" (Septiembre, 2019).

En cambio otro de los beneficios que rescata una de las madres de familia del presente estudio: "El día que hace ella psicoballet esa noche ella está como más relajada y por la tanto duerme más tranquila" (Noviembre, 2019).
En otra pregunta en correspondida con el psicoballet, la maestra expresa lo siguiente: Cuando vayan a las funciones ahí entenderán quizás el $80 \%$ o $90 \%$ de lo que es el psicoballet" (Noviembre, 2019).Otro aspecto importante es el testimonio de una madre de familia del presente estudio, señala que fue de mucha expectativa poder dialogar con los terapistas de su hija para expresarles que a su hija el psicoballet le agrada y que se compromente a trabajar mejor" (Noviembre, 2019).

Algo similar defiende la maestra del psicoballet de la actual investigación. El esfuerzo que ellos hacen en escena es tan fuerte como el que hace un niño sin discapacidad, es el mismo; obviamente quizás ellos les cueste un poquito más (Noviembre, 2019)

Ahora bien, al ser el psicoballet una terapia artística conviene mencionar lo que al Arte se le acusa, en lo referente a todo acto humano es de alguna manera comunicativo y el arte lo es en mayor medida puesto que se nutre del afán del hombre de comunicarse; este afán es a su vez originariamente una manifestación de nuestra 
dimensión de seres sociales. Así es como Bergson define la función del arte en una dirección doble, cognoscitiva y mediadora, porque según él el arte aspira a llamar directamente nuestros sentidos y nuestra conciencia, entra directamente en comunicación con las cosas. (Castro Céron, 2009).

Así mismo se hace preciso contar lo que para una danzarina aporta en su vida este Arte llamado danza, "Siento como más inspiración todos los días, me dan ganas de moverme, me dan ganas de dibujar, de saltar; de hacer cualquier cosa" (Noviembre, 2019)

A su vez alega otra bailarina, asevera que va teniendo un pensamiento más, por decirlo bailarinezco; no sé si sería así pero es un pensamiento distinto" (Noviembre, 2019)

\section{Discusión}

Afín al psicoballet, David Acosta Psic. Clínico del "Hospital de Especialidades Portoviejo" nos habla sobre la intervención que tiene la vía psiconeuroinmuneendocrinológica en esta técnica y además del beneficio en la salud que pudiese alcanzar quien lo practica y es que debido al tiempo que posee trabajando con el aspecto artístico considera que todas las artes conllevan su fortaleza, y según él; el aspecto neuropsicológico sería la fortaleza del psicoballet.

En esta investigación se concuerda con Marzo (2020), en lo referente a las terapias dancísticas, el autor plantea que se trata del uso de recursos dancísticos, artísticos que permiten a una persona con cualquier tipo de diagnósticos practicar el arte y beneficiarse de él, a través de los ejercicios rítmicos y los movimientos comenzamos a tener una restructuración en la cuestión neuropsicológica; en las funciones mentales superiores y las funciones ejecutivas.

A propósito de beneficios del que se presumen en personas de este estudio van desde la edad de 3años; vale mencionar que en la búsqueda de documentación encontramos que el psicoballet favorece también a los de edad avanzada.

También se concuerda con los resultados de Cabezas (2013) en los cuales concluye: "La autonomía al igual que la sociabilidad de los adultos mayores involucrados en el proyecto, mejoró después de la intervención psicoterapéutica, lo cual se demuestra en los resultados del postest, demostrando la validez de la terapia de psicoballet".

También se concuerda con los estudios realizados por Amador Cernuda, Director del Instituto
Universitario de la Danza "Alicia Alonso de la Universidad Rey Juan Carlos". Hace referencia a los resultados emocionales del psicoballet, indica, que son enfermos psiquiátricos y personas con alguna problemática psicológica emocional también hemos visto mejorías. También notifica que hasta el momento se han tratado a 1.700 víctimas de violencia de género. Prácticamente el 95\% ha podido superar sus problemas y ha perdido miedos" (Tejada, 2016).

Por otro lado se coincide con otras investigaciones emprendidas por Pinós Pey (2018), relata que en la última reunión anual de Society Neurocience del 2017 a la que asistieron 6800 personas prestaron mucha atención a la relación que existe entre bienestar, creatividad y danza. Los científicos recurrimos al movimiento porque este puede ayudarnos de manera multifacética a expresar emociones, trasmitir conceptos y diseñar estrategias complejas y precisas que son necesarias en el día a día.

En este sentido es importante señalar que el cerebro tiene aproximadamente 100 millones de neuronas, cada una de ellas, conectadas a miles de decenas de sinapsis. Esos átomos de pensamiento, son átomos de información, que mediante descargas energéticas se transforman en expresiones del movimiento.

De tal modo se hace conveniente mencionar, la analogía de Brown \& Parsons (2008), en la que expresa que las imágenes cerebrales revelan parte de la compleja coreografía neuronal que subyace bajo la aptitud para el baile.

Por otro lado la conexión entre danza y movimiento metafóricamente descrito por una estudiante de danza, afirma que le agrada la danza y también me gusta mucho el número ocho, entonces para mí la danza lo puedo expresar como el ocho, porque el ocho esta derecho; es como un símbolo infinito derecho como nosotras cuando bailamos, entonces es como movimientos infinitos que se puede crear no desde aquí hasta aquí sino desde donde tú quieras" (Noviembre, 2019).

Según Roger W. Simmons, de la Universidad Estatal de San Diego citado por Brown \& Parsons, (2008), afirma que cuando un bailarín de ballet pierde el equilibrio, se endereza con mayor prontitud que los no entrenados, gracias a que sus nervios y músculos responden ante las alteraciones. Un cerebro que ha aprendido danza actualiza con mayor agilidad la información que recibe su cuerpo.

En este sentido BBC News (2015), refiere que el ballet sigue siendo un espacio predominantemente 
femenino, pero a medida que los beneficios físicos del ballet se han ido conociendo más allá de los salones de clase y los escenarios, más hombres están incorporando la danza a su rutina de ejercitación [...]. Adicionar que el futbolista Rio Ferdinand se entrenó como bailarín de ballet y en Estados Unidos, es común que los futbolistas tomen clases, mientras que el equipo escocés de rugby 7 recibió entrenamiento psicológico del ex bailarín de la compañía de ballet rusa Bolshoi Misha Botting.

Es importante significar que el psicoballet integra todo lo antes planteado, acerca del ballet, la psicología, la danza y el movimiento; completándose así una terapia artística de eficaz implementación y que marca algo de diferencia entre el resto de las terapias existentes; tal y como lo afirma una madre de familia de la presente investigación, los niños no son los mismos estando aquí en el psicoballet que estando en terapia, no son los mismos.

Otras investigaciones realizadas por María Fux (citado en Barnet Lopez, 2017): “[...], plantea la danza favorece el sentirse participe y crear algo y que esto está por encima de las posibles dificultades que pueda presentar la persona" (p.47).

\section{Conclusiones}

De acuerdo a hallazgos del estudio, el criterio de expertos y la revisión bibliográfica de evidencia científica encontrada; la práctica del ballet, la danza o la experiencia del psicoballet como tal; favorecen en el desarrollo motriz tal como lo es en la postura, la resistencia, el equilibrio; a nivel anímico favorece en la autoestima, la autoconfianza, la autonomía, la relajación, aporta diversión, paz, el poder liberar emociones y a la vez controlarlas; en el aspecto social logra una mejor sociabilización y mejor desenvolvimiento. Por último, se revela también, beneficios en el aspecto neuropsicológico tanto en las funciones ejecutivas; como en las funciones mentales superiores.

Para terminar, en lo que corresponde a la población de estudio; los beneficios relatados anteriormente repercuten positivamente en las personas con y sin ningún tipo de diversidad funcional.

\section{Bibliografía}

Ballesta, A., Vizcaíno, O., Mesas, E., \& Ballesta, A. (2011). El arte como un lenguaje posible en las personas con capacidades diversas. Arte y Políticas de Identidad, 4, 137-152. Recuperado

de https://revistas.um.es/reapi/article/view/14 6051/130461

Barnet Lopez, S. (2017). Implementación de un programa de danza para personas con Discapacidad Intelectual: Repercusiones en su Bienestar Emocional (tesis doctoral). Universidad Ramon Llull, Barcelona, España.

BBC NEWS. (10 de mayo de 2015). Lo que el ballet puede hacer por tu cuerpo. Recuperado de https://www.bbc.com/mundo/noticias/2015 /05/150427_deportes ballet_ejercicio_salu d finde dv

Brown, S., \& Parsons, L. (2008). Neurociencia de la danza. Investigación y Ciencia, 84-89. Recuperado de https://dantzan.eus/fitxategiak/2008-0901_Investigacion-y-ciencia-Neurocienciade-la-danza.pdf

Cabezas -Zabala, G. (2013). Eficacia del psicoballet aplicado como terapia grupal en la mejoría de la autonomía personal de los adultos mayores (trabajo de posgrado). Universidad Central del Ecuador, Quito, Ecuador.

Campos, C. (2015). Trastornos respiratorios en niños con síndrome de Down. Neumol Pediatr, 10, 101-105. Recuperado de https://www.neumologia-pediatrica.cl/wpcontent/uploads/2017/07/trastornosrespiratorios.pdf

Castro , M. . (2009). El carácter plástico de las formas de notación: poesía, música y danza (tesis doctoral). Universidad Complutence de Madrid, Madrid, España.

Fariñas-García, G. (2011). Psicoballet; ciencia y arte. Recuperado de: https://books.google.com.ec/books? $\mathrm{id}=\mathrm{OCa}$ vhuw2eQ4C\&printsec $=$ frontcover $\& \mathrm{dq}=\mathrm{ge}$ orginafariñas\&hl=es419\&sa=X\&ved=0ah UKEwisifiUp83oAhUIVN8KHSUOCgYQ $\underline{6 A E I J z A A \# \mathrm{v}=\text { onepage } \& \mathrm{q}=\text { georginafariña }}$ $\underline{\mathrm{s} \& \mathrm{f}=\text { false }}$

Gómez, S., Jaimes, V., Palencia, C. , Hernández, M., \& Guerrero, A. (2013). Parálisis cerebral infantil. Archivos Venezolanos de Puericultura y Pediatría, 76, 30-39. Recuperado de https://www.redalyc.org/pdf/3679/367937 046008.pdf 
Grupo ROP Argentina, \& Ministerio de salud. (2016). Guía de práctica clínica para la prevención, diagnóstico y tratamiento de la retinopatía del prematuro (ROP). Recuperado de http://www.msal.gob.ar/images/stories/bes/ graficos/0000000723cnt-guia-rop-2016.pdf

Pinós Pey, K. (2018). Neurociencia y la danza. Recuperado

de https://www.uv.mx/danza/files/2018/03/Ne urociencia-y-la-danza-2.pdf

Quecedo, R., \& Castaño, C. (2003). Introducción a la metodología de investigación cualitativa. Revista de Psicodidáctica, 7, 5-39. Recuperado de https://www.redalyc.org/pdf/175/1750140 2.pdf

Rey-Gónzales, A., \& García-Blas, B. (2018). Cómo abordar el Trastorno del Espectro del Autismo desde los medios de comunicación. Recuperado de http://www.autismo.org.es/sites/default/file s/como-abordar-el-trastorno-en-losmedios.pdf

Romañach, J., \& Lovato, M. (2005). Diversidad funcional, nuevo término para la lucha por la dignidad en la diversidad del ser humano. Recuperado http://forovidaindependiente.org/wpcontent/uploads/diversidad funcional.pdf

Tejada, M. (2016). Con la danza niños hiperactivos han dejado de tomar pastillas. Recuperado de

https://www.laeducacioncuantica.org/educ acioncuantica/SEducacionCuantica?PN=1 $\underline{6 \& \mathrm{PE}=2 \& \mathrm{WEBLANG}=1 \& \mathrm{VOLNOT}=\text { noti }}$ cia6\&VOLTIP=-

$1 \& \mathrm{VOLPAG}=1 \&$ NOTICIA=447. 